\title{
Unstated Premises
}

\section{MICHAEL BURKE University of Indiana at Indianapolis}

\section{Introduction}

Many arguments rest on unstated assumptions. These include assumptions which underlie stated premises and assumptions which underlie inferences from stated premises to conclusions. Consider the argument below.

\section{This wine can't be Port. If it were, it would be sweet.}

That Port is sweet might be an unstated assumption underlying the stated premise. That this wine is not sweet might be an unstated assumption underlying the inference. In Robert Ennis' suggestive terminology, the first is a "back-up," the second a "gap-filler." Having now acknowledged back-ups, I will hereafter ignore them. When speaking of "unstated assumptions" or "unstated premises," I will always mean the gap-filling variety.

When we set out to identify an argument's unstated premises, we should be clear whether our purpose is (1) to identify the argument intended by the arguer or (2) to determine whether the stated premises provide the makings of an acceptable argument for the conclusion.[1] Although this distinction is now frequently noted, few of those who provide "criteria for unstated premises" bother to provide two sets. And few provide separate discussions of what really are quite different enterprises.

One who does is Robert Ennis [p. 62], who distinguishes "needed assumptions" ("propositions that are needed to support the conclusion, to make the argument a good one...") and "used assumptions" ("unstated reasons that a person actually used consciously for subconsciously, if you believe in subconscious reasons) as a basis of argument..."). That a certain assumption was used, Ennis argues, is best understood as an explanatory hypothesis. The argumentative utterance constitutes an explanandum. And we should attribute to the arguer whatever unstated assumption best explains, given what we know about the arguer and the context, the details of the utterance. Ennis' criteria for used assumptions are simply an application of the general criteria for evaluating singular explanatory hypotheses.

As best I can judge, Ennis' treatment of used assumption is successful, so far as it goes, and a helpful contribution to the subject of unstated premises. Less satisfactory, in my opinion, are his and other writers' accounts of "needed" assumptions, and it is on these that I will focus throughout this paper. More precisely, I will be concerned with criteria for identifying unstated premises when our purpose is to determine whether stated premises provide the basis for an acceptable argument for a given conclusion. After explaining my dissatisfactions with the accounts of Ennis, Hitchcock, Schwartz and Scriven, I will offer an alternative. First I will note (but not try to make precise) two criteria which are uncontroversial.

\section{Uncontroversial Criteria: Sufficiency and Preservation}

Most writers recommend that we identify as an argument's unstated premises propositions that would combine with the stated premises to make the argument deductively valid. This may reflect not a general commitment to deductivism, but simply the tradition 
of discussing unstated premises in connection with "deductive" arguments. Some writers, however, require only that the unstated premises strengthen the inference, that the conclusion be more probable relative to the stated premises plus the unstated ones than relative to the stated premises alone. The stronger requirement might be defended by arguing, as Thomas Schwartz does [1981, p. 245], that "any argument can be construed as deductive without impairment." But what is uncontroversial is what 1 will call the "sufficiency requirement": The unstated premises must be sufficient to validate, or at least to strengthen, the inference.

But now for any deductively invalid inference there are infinitely many sets of propositions whose addition would validate or strengthen the inference. How are we to choose? One justly popular criterion is what I will call "preservation," according to which we should try to preserve the role of the stated premises. So long as our purpose is to evaluate the strongest argument that can be based on these premises, we will not want to add propositions that would nullify their contribution to the strength of the inference. Otherwise the resulting argument would not be based on the stated premises. But even when we combine sufficiency with preservation, we will always still be left with an infinite number of candidates. (Disjoining the negation of the conjunction of the stated premises with any statement that entails the conclusion will produce such a candidate.)

\section{Section I: Ennis}

To the criteria of sufficiency and preservation (for which he uses other names), Ennis adds "fidelity" and "plausibility."

Ennis' fidelity is not the criterion familiar in discussions of used assumptions, i.e., fidelity to the beliefs and intentions of the arguer, but what Ennis calls "fidelity to the argument."
And as is plain from Ennis' brief explanation [p. 70], fidelity to the argument is not so much a criterion for unstated assumptions as an injunction (to apply when the argument, rather than the conclusion, is "the focus" ${ }^{\prime \prime}$ ) against revision of the stated parts of the argument. It will not be relevant to our concerns.

Ennis' fourth criterion is plausibility. The essentials of his explanation are contained in these passages [p. 71]:

\begin{abstract}
The plausibility criterion calls for the assumption identifier to be as generous as possible. One does this (selecting from the possible gap fillers) by attributing to the argument or conclusion maximally-plausible propositions. The reason for this is that it is not fair to say that a less plausible proposition is needed by a position, when a more plausible one is available to do the job of gap filling... Since there is...sometimes a choice between attributing one broad one and two more-narrow ones, the criterion, more fully stated, calls for attributing assumptions with a maximal joint plausibility.
\end{abstract}

There is a problem. Initially to oversimplify, the problem is that the most plausible candidate will always be the weakest candidate. And if ' $\mathbf{P}$ ' abbreviates the conjunction of the premises of some deductively invalid argument, and ' $C$ ' its conclusion, the weakest validating proposition is expressed simply by "If $\mathbf{P}$ then $\mathbf{C}$ '. (Note that this candidate will violate neither preservation nor Ennis' fidelity.) I will call this the "reiterative" candidate, since several writers remark that it merely "reiterates" the arguer's claim that the conclusion follows from the premises. Although the reasons differ, and are not always clear (I'll give mine later), few writers think it helpful to add this "logical minimum." More precisely, few, if any, think it generally to be the best choice.

Oddly, Ennis takes no notice of this problem, even though he does mention reiterative tacit premises. Ennis notes that he differs with Scriven in holding that these minimum links sometimes are a useful addition, but he doesn't 
explain how, on his criteria, we ever could justify a preference for one of their validating competitors.

But now am I right in saying that the weaker of two claims cannot be the less plausible? After three points of clarification, I believe the reader will be ready to agree.

First, I am using 'weaker' in its standard logical sense: $\mathbf{P}$ is weaker than $\mathbf{Q}$ if and only if $\mathbf{Q}$ entails, but is not entailed by, $\mathbf{P}$. Ralph Johnson [p. 7] suggests that on occasion the "stronger" candidate may be the more plausible, but he is using 'stronger' in a different sense. Comparing 'Most redheads are quick-tempered' with 'Most redheaded women are quicktempered', Johnson finds the former to be stronger, in an "as-yet unanalyzed" sense, and also more plausible. But since neither statement entails the other, neither is stronger in the standard logical sense. Johnson does not suggest that in this sense of 'stronger', the stronger of two claims might be the more plausible.

Second, I am not denying that it may often be more plausible to hold that an arguer had in mind the stronger claim. With reference to the same example discussed (later) by Johnson, Scriven [p. 166] says that "it's a little more plausible to suppose that the arguer believes that red-headed people are bad-tempered than that he or she believes that red-headed women are bad-tempered..." Perhaps so. But, of course, its being more plausible that a certain assumption was made is quite different from its being more plausible that that assumption is true.

Third, my claim that the weaker of two propositions is never the less plausible is meant to carry the implicit qualification: providing its relative weakness is realized. The entailments of a proposition sometimes are less plausible to us than the proposition itself, until we notice that they are entailments. That a purple shoe can be evidence that all ravens are black is wildly implausible-initially, but it is a logical consequence of three principles each of which is extremely plausible-initially.
Once we are acquainted with the raven paradox we revise our assessments. We consider the (conjunction of) the principles no more plausible than their surprising consequence. Now when deciding which of two assumptions to identify as the assumption needed by an argument, should we select (other things being equal) the assumption that had the greatest initial plausibility - or the one we find most plausible when our reflections have concluded? Given that Ennis says, "The plausibility criterion calls for the assumption identifier to be as generous as possible... The idea is to give the argument or conclusion its best chance to succeed..." [p. 71], he could hardly suggest that we always select the candidate with maximum initial plausibility. Sometimes we find decisive objections to that candidate while discovering strong support for one of the others. But if we are to choose on the basis of final plausibility, then since we realize that the reiterative candidate is entailed by any of its validating competitors, the plausibility criterion will always be powerless to justify our preference for one of those competitors.

Ennis seems tacitly and inadvertently to concede the point when he introduces the one reiterative candidate he discusses by saying [p. 75], 'But I can note a minimal coverage maximallyplausible claim that would serve as a piece in a reconstructed version of the Will argument..." The only apparent ground for regarding the introduced claim as "maximally-plausible" is precisely its "minimal coverage." Subsequently, Ennis remarks [p. 75], "Since we are not operating in a real context, I cannot suggest whether the request should be to consider only the specified claim or a more general one." He seems not to have thought to ask how his criteria could warrant a preference for the more general claim.

In sum: Since it is not apparent how plausibility or any of Ennis' other criteria can enable us ever to reject the reiterative candidate in favor of one of its stronger competitors, and since, as we will see, there are good reasons for thinking such competitors sometimes to 
be preferable, I conclude that Ennis' account is inadequate.

\section{Section II: Hitchcock}

One of the more active contributors to the theory and pedagogy of informal logic is David Hitchcock, whose generally excellent textbook includes a relatively thorough treatment of tacit premises. The policy he recommends is to add the weakest candidate satisfying validation, preservation, and "testability." His strategy for blocking the reiterative candidate is contained in these passages:

Any argument can be given a sound inference by adding as a tacit premise that if the explicit premises are true, the conclusion is true. In general, however, such a tacit premise is not testable... The rationale for the criterion of testability is that in supplying a set of tacit premises for an argument we are trying to see whether a good case can be made for the conclusion. If we put in a statement that is not testable, then, when we get to the stage of assessing whether we are warranted in believing the statement, we have to think about how it could be shown to be true (or probably true). But unless there is some way of testing it, this is impossible.

Consider the argument "He's a homosexual, so he shouldn't be appointed to this politically sensitive post." The weakest tacit premise which will make the conclusion follow is the conditional statement: "If he's a homosexual, he shouldn't be appointed to this politically sensitive post." But this statement is not testable; it merely reiterates the claim of the argument that it follows from his being a homosexual that he shouldn't be appointed. We need a more general statement; for example, "Homosexuals ought not to be appointed to politically sensitive posts." This is a principle; one could ask for its justification (a justification which would presumably point to some link between homosexuality and political sensitivity) and also argue against it (on the ground, for example, that sexual orientation should be irrelevant to hiring decisions and that $a$ responsible political organization should show leadership in this respect). [p. 83]
Hitchcock does not give a definition of 'testable'. He is content to say that testability may be either "direct or indirect" and that it need not be empirical. The testing may be "in terms of our usage of a certain word... of a mathematic theory... of a normative theory of ethics or aesthetics..."[p. 83].

Now I think we can accept both the criterion of testability and the rationale offered for it. But will this criterion exclude reiterative tacit premises? Will it, for example, exclude the statement, "If he's a homosexual, he shouldn't be appointed to this politically sensitive post," call it ' $\mathbf{S}$ ', that Hitchcock uses it to exclude?

It is clear, I believe, that the answers are "no." But let's look at Hitchcock's reasons for saying otherwise. First, he notes that $\mathbf{S}$ "merely reiterates the claim of the argument that it follows from his being a homosexual that he shouldn't be appointed." That's true, but what reason is there to think the latter claim untestable? Second, Hitchcock contrasts $\mathbf{S}$ with the statement, call it 'P'. "Homosexuals ought not to be appointed to politically sensitive posts." Hitchcock says of $\mathbf{P}$ that "one could ask for its justification... and also argue against it." But one could ask for the justification of $\mathbf{S}$. In general, considerations which support $\mathbf{P}$ will support $\mathbf{S}$. One could also argue against $\mathbf{S}$. One could use the premises Hitchcock suggests for arguing against P.

To see the futility of Hitchcock's approach, consider this. Even if $\mathbf{S}$ is understood as a material conditional, $\mathbf{S}$ will be sufficient to validate Hitchcock's argument. Now if $\mathbf{S}$ is so construed, it will be equivalent to "Either he's not a homosexual or he shouldn't be appointed to this politically sensitive post." And it seems safe to say that this (material) disjunction is untestable only if at least one of its disjuncts is untestable. So if $\mathbf{S}$ is untestable, and therefore ineligible ever to serve as an unstated premise, so is at least one of these disjuncts. Furthermore, if $\mathbf{S}$ and one of the disjuncts are untestable, they would also be unsatisfactory as stated prem- 
ises or as conclusions. These consequences are unacceptable.

More generally, one would think that a given reiterative tacit premise is testable if the inference which it validates is testable. If so, then if reiterative tacit premises are, "in general," untestable, so are formally invalid inferences.

In light of the above, it is plain that Hitchcock's policy - to add the weakest candidate satisfying validation, preservation, and testability-generally will select the reiterative candidate. In seeking to exclude this candidate by reference to an intrinsic defect such as untestability, Hitchcock overlooks the fact that a statement which is unsuitable to serve as one argument's tacit premise may serve quite satisfactorily as another's conclusion or explicit premise (or even as another argument's tacit premise). What Hitchcock could have said is that argument evaluation is never facilitated by adding the reiterative statement, because it is never easier to test this statement than to test the original inference. (Of course, it doesn't follow that we would be justified in adding some stronger statement. All that follows is that we might as well add nothing. Hitchcock would still need to provide and justify additional criteria.)

\section{Section III: Schwartz}

One of the very few writers with a criterion capable of blocking the reiterative candidate is Thomas Schwartz. To the familiar criteria of validation, fidelity (which he says to be important only when the aim is to identify the argument intented by the arguer), and plausibility (which he calls "Generosity"), Schwartz adds "Generality": "Add premises that are as general as possible, consistent with Fidelity and Generosity" [1981, p. 236]. The rationale for this criterion is explained thus:

Say your are deciding which of two [validating] candidate premises to add to an argument, and neither is preferable according to Fidelity or Generosity [plausibility], but one is more general than the other. The reason you should choose the more general candidate is that it is a likely reason or justification for the less general one, hence a likelier ultimate reason or justification for the argument's conclusion-a likelier premise, in other words [1981, p. 236].

What we would need to understand, before we could accept Schwartz's account, is why, when the purpose in identifying unstated premises is to make the argument as strong as possible, generality should be a desideratum. Ordinarily, an argument's strength is thought to depend on the plausibility of its premises, on the strength of the link between its premises and conclusion, and on the absence of circularity. Neither of the first two factors can provide a rationale for the generality criterion. This criterion is to apply only when plausibility and validation yield no decision. And there is no apparent way in which the criterion can be justified by reference to the need to avoid circularity.

Schwartz's rationale, surely unaceptable, is based on the idea that only an "ultimate reason" (basic proposition?) is a genuine premise. This idea is supported by neither the ordinary, the legal, nor the logical usage of "premise," and it is inconsistent with most of Schwartz's own examples of stated and tacit premises. It also conflicts with Schwartz's statement, found on the following page [1981, p. 237], "'Since any statement about any subject could be premise of an argument, you would be omniscient if you could tell the truth or falsity of every premise of every argument you might ever come across." And even if we agreed to the narrow definition, this would merely shift the question to why an argument's undefended parts must be limited to premises so defined.

I suspect that the true appeal for Schwartz of the generality criterion is that he thinks it will facilitate (fair) criticism. For stated premises, Schwartz proposes a "test of Sufficient Generalitv."

Is there some natural way to make the premise in question more general...? 
If so, ... is there some relevant difference between the cases to which the premise already is applied and the others in the wider class - some difference that would justify applying the premise to the former cases only? If not, the premise fails the Sufficient Generality test... the author is committed to a more general premise... The test... is useful as a critical tool because the more general of two statements often is the easier to criticize... one cannot immunize one's premises from counter-examples by restricting their generality unless there is a plausible reason for the restriction. So if there is no plausible reason not to generalize a premise a certain way, a counterexample to the generalized version refutes the original. [1980, p. 188]

Without commenting directly on the merit of these ideas for dealing with stated premises, I wish to ask whether they can justify a generality criterion for unstated premises. Suppose that $\mathbf{A}$ and $\mathbf{B}$ are the leading validating candidates, that $\mathbf{B}$ is more general than $\mathbf{A}$, that we see no reason for the restriction contained in $\mathbf{A}$, and that we consider $\mathbf{B}$ to be about as plausible as $\mathbf{A}$. Now why should we choose B? Suppose that in accordance with the generality criterion, we proceed to identify $\mathbf{B}$ as the tacit premise. And suppose that subsequently we discover a counterexample to B (one which is not a counterexample to A). Suppose, finally, that we continue to see no reason for the restriction contained in $\mathbf{A}$ (except for its ability to block the counterexample). Now what should we do? Should we replace B with A? Or can we properly conclude our analysis, charging that the argument depends on an assumption shown to be false? (If we can, then just criticism has indeed been facilitated by use of the generality criterion.) Evidently Schwartz would have us retain B. Schwartz says that "one cannot immunize one's premises from counterexamples unless there is a plausible reason for the restriction." (I take it that he means "unless one has a plausible reason for the restriction.") But this is inconsistent with the plausibility criterion, which is supposed to take precedence over the generality cri- terion. If we are now able to refute $\mathbf{B}$, then $\mathbf{B}$ is now significantly less plausible than $A$-unless we are able also to refute $\mathbf{A}$. And to refute $\mathbf{A}$ it would not be sufficient to point to the refutation of $\mathbf{B}$ plus the absence of any apparent reason for the restriction contained in A. It would be necessary to show that no such reason exists, i.e., that $\mathbf{A}$ is false if $\mathbf{B}$ is. Short of that, our final assessment should be that the argument depends on a tacit premise, A, which there is no apparent reason to accept. That accords better with our purpose (evaluating the strongest argument that can be based on the stated premises) than concluding that the argument depends on a tacit premise, B, of demonstrable falsity. The plausibility criterion will not permit us to continue to identify $\mathbf{B}$ as the tacit premise when we have come to have a stronger reason for rejecting $\mathbf{B}$ than for rejecting $\mathbf{A}$. The "immunization" not only would be permissible; it would be obligatory. So how, consistently with the plausibility criterion, could just criticism ever be facilitated by use of the generality criterion? If there is an answer, Schwartz has not provided it.

To sum up: Although Schwartz's generality criterion has the (presumed) merit of frequently rejecting the reiterative candidate, its rationale is obscure. There is no apparent way to justify it by reference to the aim of making the argument under analysis maximally strong. No candidate can have greater (final) plausibility than the reiterative candidate. Schwartz's stated rationale - that the more general candidate is a likelier ultimate reason and, therefore, a likelier premise-depends on the unacceptable notion that only ultimate reasons are genuine premises. Finally, it seems futile to seek to justify the criterion by reference to the aim of facilitating just criticism. If we should ever come to have a stronger reason for rejecting the more general candidate than for rejecting the less general one, the plausibility criterion would require that we then designate the less general candidate as the tacit premise. 


\section{Section IV: Scriven}

Michael Scriven's highly-regarded textbook Reasoning contains unusually thorough coverage of what Scriven calls "unstated assumptions" or "missing premises." It is not easy, however, to extract a coherent account.

On the one hand, Scriven wants us to look for an unstated link that is "plausible" [pp. 174-175], "true" [p. 174], "obviously" assumed [p. 83] and "persuasive or at least not objectionable in and of itself"

On the other hand, Scriven says, "Your task... is to call attention to... the important, the debatable, the hazardous, and the hidden points... In short, you're searching for a weak link..." [p. 164].

Scriven notes that there is "some tension" among his various desiderata. But between "obviously assumed" and "hidden," and between "a weak link" and "not objectionable in and of itself," tension has escalated into outright contrariety.

Part of the confusion, as I see it, stems from Scriven's failure to give separate accounts of used assumptions and what he calls "required" assumptions [p. 81]-or else to limit his account to one or the other. Scriven writes:

\begin{abstract}
You also want to try to relate the assumptions as you formulate them to what the arguer would be likely to know or would believe to be true. The principal function of argument analysis is not that of reconstructing the state of mind or body of beliefs of the arguer, but this may nevertheless be a relevant consideration in some cases. You need to decide whether you are arguing against the arguer or the argument. [p. 85]
\end{abstract}

Regrettably, Scriven does not go on to provide separate accounts of "arguing against the arguer" and "arguing against the argument."

Also contributing to confusion, I believe, is a failure to keep separate two questions that Scriven himself distinguishes: (1) What unstated propositions does an argument (or arguer) assume? (2) Which of an argument's (or arguer's) unstated assumptions are worth identifying as such? Most discussions of enthymematic arguments focus on the first question. Since Scriven has (as we will see) a rather broad conception of "unstated assumptions," he understandably gives much attention to the second. Indeed, the second of his two sections on unstated assumptions is titled "Significant and Insignificant Assumptions." The significant assumptions, those worth identifying, are the "important," "illuminating," "debatable," "vulnerable," "hazardous," or "hidden" ones. I suspect that Scriven's other criteria ("plausible," "not objectionable," "obviously assumed") are given with the first question in mind, especially as concerns used assumptions. But Scriven never raises the first question without proceeding to conflate it with the second [cf. pp. 85-86], and it is not obvious how he would answer it. [2]

The value in Scriven's treatment is found, I believe, in his illustrations, the most extended of which concerns the argument below.

\section{He is a homosexual. \\ Hence, he is prone to blackmail. \\ Hence, he is a security risk. \\ And, this job requires security clearance. \\ So, he should not be appointed.}

Scriven begins by rejecting as "at too trivial a level" [p. 169] candidates like "Homosexuals are prone to blackmail" and "Male homosexuals are prone to blackmail." After noting a couple other "obvious" and "unilluminating" assumptions, Scriven identifies three "illuminating" ones:

So the first serious problem [my emphasis] with the argument is that it assumes that the individual is not already known to be a homosexual. [p. 171]

So the argument makes the weak assumption [my emphasis] that everyone would rather pass up a job of this type 
than [publicly] acknowledge homosexuality [as a condition of appointment]. And that's something that wasn't obviously assumed. [p. 171]

So the argument, in its general form, does rest upon an assumption... [namely] the proportion of homosexuals in communities which would be powerful enough to have them removed from their jobs is greater than the proportion of heterosexuals in communities which would react just as unfavorably to heterosexual "misdemeanors." And it isn't at all clear that the reverse isn't the case... That's an interesting discovery about the argument and a real weakness in it. [p. 172, my emphasis]

What Scriven seems to be after, when analyzing an argument, are the most implausible propositions that the argument truly "assumes" or "depends on." (Scriven uses these terms interchangeably.) The aim is to test the argument by seeing whether it can be shown to depend on implausible assumptions. Of course, this raises, urgently, the first of the two questions I distinguished earlier: How can we tell whether a given inference assumes a given proposition? Unfortunately, Scriven never answers this question. The answer suggested by his examples is something like this: An inference assumes (depends on/depends on the assumption of) proposition $\mathbf{A}$ (however plausible or implausible $\mathbf{A}$ may beand however weak or strong) just in case the inference is justified only if $A$ is true.

Now I have no objection to Scriven's method of evaluating arguments. My objection is to his characterization of the method. I will argue that Scriven's procedure, contrary to its billing, is not one of identifying and evaluating missing premises, but rather one of evaluating inferences without identifying missing premises. Scriven himself makes the distinction. Near the beginning of his discussion, he writes [p. 81]:

...this illustrates a very general principle about argument analysis. You can either leave the argument the way you find it, in which case a good deal of your criti- cism will be criticism of the inferences in it; or you can patch it up by adding some assumptions on which it is obviously depending, in which case the inferences will be pretty satisfactory, but the assumption will now come under fire.

Now as Scriven himself remarks [p. 170, p. 171], the "assumptions" he identifies in the homosexual argument are not ones on which the argument is "obviously depending." The obvious candidates had been spurned as "at too trivial a level" [p. 169]. So one might expect Scriven not to conceive the three "assumptions" as unstated parts of the argument, but rather to conceive their denials as premises of his criticism of the inference.

Now despite the scare quotes I used in the last paragraph, I do agree that there is a sense of 'assumes' in which the homosexual argument does indeed assume (or might be argued to assume) the three implausible propositions Scriven says it assumes. This is the sense in which an argument may be said to assume any unstated proposition on which its inference depends, meaning any proposition which needs to be true, if the inference is to be justified. But is just any such proposition a "needed assumption," in the sense intended by Ennis, Hitchcock, and Schwartz? No. A needed assumption, in this latter sense, is one which needs to be added, or (better) needs to be a premise of the argument, if the argument is to be as strong as it can. That is, it needs to be included among the propositions from which the conclusion is inferred. Now there are infinitely many propositions which need to be true if an inference is to be justified. (Examples: that there is at least one inference; that there is at least one justified inference; that there is at least one thing that is either an inference or a polar bear.) Very few of them will be needed to serve as supplements to the stated premises. That is, very few of them are needed assumptions, in any sense in which a needed assumption is a needed premise. And Scriven has given no reason for thinking that 
the homosexual argument, if it is to be as strong as it can be, needs to contain as premises the three implausible assumptions which do (arguably) need to be true if its inferences are to be justified.

In short, we should distinguish (1) used unstated assumptions, i.e., the unstated propositions actually assumed by the arguer, (2) needed unstated assumptions, i.e., the unstated propositions that need to be included among the propositions from which the conclusion is inferred, if the argument is to be as stong as possible, and (3) the unstated assumptions on which the argument depends, i.e., the unstated propositions which need to be true, if the argument's inference is to be justified. Our concern in this paper is with unstated assumptions of the second sort. In chapters titled "Unstated Premises," authors of informal logic texts generally are concerned with assumptions of the second or first sort. Only these assumptions are properly considered parts of the argument or premises. [3] Searching for the most implausible assumptions of the third sort is best conceived as evaluating the inference without first identifying missing parts $-a$ procedure that may often be eminently suitable.

In sum: I suggest that we take Scriven not to be offering criteria for unstated assumptions, in any sense in which an unstated assumption is a premise. What Scriven demonstrates is a method of evaluating inferences without first identifying missing parts The method is that of seeing whether the inferences can be shown to depend on implausible propositions. (The linguistic acceptability here of "assumptions" is what can mislead one into thinking that Scriven is looking for the same sort of thing as Ennis, Schwartz, and Hitchcock.) To complete his account, Scriven needs to say what it is for an inference to "depend on" a proposition. (I have offered a suggestion.) He may also wish to draw upon his neglected weakness, plausibility, unobjectionability, and obviousness criteria for an account of used or need- ed unstated premises. (As will soon be clear, I don't consider this urgent.) When Scriven deals separately with (1) used unstated assumptions, (2) needed unstated assumptions, the assumptions on which an inference depends, and (4) those of the latter which are worth identifying as such, much of the "tension" will be relieved.[4]

\section{Section V: Outline of a Policy}

Finally, I will outline my own ideas on unstated premises. These ideas are meant to apply when our aim is to determine whether the stated premises provide the basis for an acceptable argument for the conclusion, not when our aim is to identify the argument intended by the arguer.

As noted by Scriven and others, it is always possible to evaluate an argument without first identifying unstated premises. We can instead evaluate the stated premises and the inferences. We can evaluate the inferences by seeing, à la Scriven, whether we can think of any implausible propositions on which the inferences depend-in the sense of "depend" identified in the preceding section. Why, then, might we wish to search for unstated premises?

The answer, I think, is supposed to be something like this. If we can find unstated propositions which (1) satisfy preservation, (2) suffice to validate, or at least to justify, the inference, and (3) are themselves acceptable, then we will have found reason for judging the original inference acceptable. If, on the other hand, we can find no such propositions, and if we find a proposition which we have reason to reject but which we can show to be needed by the argument, in the sense that this proposition must be regarded as an unstated premise if the argument is to be as strong as it can be, we will then be able to criticize the argument by giving our reason for rejecting this proposition.

My view, which I will defend below, 
is that only when we ourselves accept a proposition is it useful to identify it as an unstated premise. If we find no acceptable candidate satisfying sufficiency and preservation (the two uncontroversial criteria discussed earlier), then we should evaluate the inference without identifying unstated premises.

If we search for an acceptable set of propositions satisfying sufficiency and preservation, one of three things will happen: (1) We find just one; (2) we find more than one; (3) we find none.

(1) If we find just one acceptable set of propositions (satisfying sufficiency and preservation), then we may identify these propositions as unstated premises. The reason for doing so is to make explicit (for ourselves or for an audience) why we accept the original inference. If we think others may have doubts about the identified propositions, we may wish to give our reasons for accepting them. There is more point in doing this, of course, when we find the rest of the argument acceptable as well.

(2) If we find more than one acceptable set (satisfying sufficiency and preservation), then we should be guided by whatever further criteria guide our choice of stated premises when we are constructing an argument. (In effect, we are constructing an argument-an argument for accepting the original inference.) Evidently there is little felt need for the identification of these criteria, and I will be content to suggest a couple of possible considerations: (a) and (b).

(a) If our choice has narrowed to candidates $\mathbf{A}$ and $\mathbf{B}$, and if $\mathbf{B}$ is more general than $\mathbf{A}$, and if our only reasons for accepting $A$ are our reasons for accepting $\mathbf{B}$, then probably we should pick B. Otherwise we may mislead our audience concerning our reasons for accepting $\mathbf{A}$. To assert that jogging is dangerous for tall women with blue eyes is to imply that one's evidence provides significantly less support for various wider generalizations.

(b) A weaker candidate, especially the reiterative candidate, will some times have less initial plausibility than some stronger candidate. (In the case of the intentionally mystifying enthymemes of Sherlock Holmes, the plausibility of the reiterative candidate is initially nil.) When the stronger candidate is more plausible initially, and about as plausible finally, adding the stronger may be dialectically more efficient. We may avoid having to support the added premise.

(3) If we find no acceptable proposition (satisfying sufficiency and preservation), I believe we should evaluate the argument without identifying unstated premises. Actually, I would allow one trivial exception: We may add the reiterative candidate. This is always unobjectionable, since the acceptability of the reiterative candidate is a necessary condition of the acceptability of the argument, but never useful, in regard to facilitating evaluation, since it is never easier to evaluate the reiterative candidate than to evaluate the corresponding inference.

I will now explain why I believe we should add no proposition unacceptable to us other than the reiterative candidate. My procedure will be to compare adding the reiterative candidate, $\mathbf{A}$, with adding some other candidate, B (when we accept neither A nor B). This will be verbally less cumbersome than comparing adding nothing with adding some condidate other than the reiterative one. And, of course, adding and evaluating the reiterative candidate is equivalent to adding nothing and evaluating the inference. But since the latter strategy is one step shorter, it is the latter that should be simpler to employ, although it is the former that will be easier for me to talk about.

(a) Suppose, then, we are deciding whether to add the reiterative candidate, A, or some stronger candidate, B. And suppose that we accept neither A nor $\mathbf{B}$. Now either we have a stronger criticism of $\mathbf{B}$ than we have of $\mathbf{A}$, or we do not.

(i) If we do not, then, of course, we should add and criticize $A$. We will then have criticized the weakest assumption capable of validating the argument. 
If we added and criticized $\mathbf{B}$, we would only have to go on to discuss whether we might accept some weaker, though still validating proposition. Of course, it may be that we will criticize $A$ by criticizing $\mathbf{B}$-and arguing, asserting, or at least implying that there is no other reason for accepting $\mathbf{A}$. Even if so, when our criticism of $\mathbf{A}$ is as strong as our criticism of $\mathbf{B}$, we will want to make clear that we are attributing to the argument only the weakest validating assumption and that the point of our criticism of $\mathbf{B}$ is to challenge the only support for that assumption.

(ii) If, on the other hand, we do have a stronger criticism of $\mathbf{B}$ than of $\mathbf{A}$, then our aim of evaluating the strongest argument that can be based on the stated premises dictates that $\mathbf{A}$ be identified as the unstated premise. If, for example, we can refute $\mathbf{B}$ but not $\mathbf{A}$, and if we can claim no more than that $\mathbf{B}$ is the only apparent reason for accepting $\mathbf{A}$, our assessment should be not that the argument depends on an unstated premise, $\mathbf{B}$, of demonstrable falsity, but rather that it depends on an unstated premise, A, for which there is no apparent justification.

(b) Suppose, finally, that the two unacceptable finalists are the reiterative candidate, $\mathbf{A}$, and a non-validating candidate, $\mathbf{B}$. If we have a stronger criticism of $\mathbf{B}$ than of $\mathbf{A}$, then since $\mathbf{A}$ is all that the argument needs, we should add and criticize $\mathbf{A}$. If our stronger criticism is of $\mathbf{A}$, then since the acceptability of $\mathbf{A}$ is a necessary condition of the acceptability of the argument, again we should add and criticize A.

In summary: When our purpose is to determine whether stated premises provide the basis for an acceptable argument for a given conclusion, I recommend the following policy. If there are unstated propositions satisfying preservation and sufficiency that we ourselves accept, we may identify them as unstated premises. The reason for doing so is to make explicit (for ourselves or for an audience) why we accept the original inference, If we find two or more acceptable sets of propositions (satisfying sufficiency and preservation), then we should choose on the basis of whatever further criteria guide our choice of stated premises when we are constructing an argument. (In effect, we are constructing an argument, an argument for accepting the original inference.) in part $2 \mathrm{~b}$ of this section, 1 indicated the basis on which we may often reject the reiterative candidate in favor of one of its (also acceptable) competitors. If we find no acceptable candidate (satisfying sufficiency and preservation), I believe we should evaluate the inference without identifying unstated premises (or, which amounts to the same thing, add and evaluate the reiterative premise). My reasons were given in part 3 of this section.

To conclude: In the five sections of this paper, each of which concludes with a summary, I have criticized the unstated-premise policies of Ennis, Hitchcock, Schwartz, and Scriven; and I have outlined an alternative. [5]

\section{Notes}

[1] Van Eemeren and Grootendorst have suggested that our target should be the unstated propositions to which the speaker is committed by his argumentative speech-act. Viewing unstated premises as Gricean conversational implicatures, they write: "It is not what the speaker himself may think he has left unexpressed, but what he can be held to by the listener, that must count $^{\prime \prime}$ [p. 220]. Now attention to conversational implicatures may yield important evidence of the arguer's intentions. And if we are able to engage the arguer in dialogue, pointing out these conversational implicatures may help elicit the arguer's true intentions. But unless we are engaging in rhetoric, or else in an academic exercise, our ultimate goal will be to identify and evaluate either the intended argument or the strongest argu- 
ment, not the argument to which the speaker, regardless of his intentions, has "committed" himself.

[2] His frequently repeated "strong enough, but no stronger than necessary" formula [p. 85] doesn't help here. The reiterative candidate is counted as an assumption [p. 163], so it must be "strong enough" to be an assumption. But any stronger proposition would then be "stronger than necessary" to be an assumption. Yet some stronger propositions are so counted.

[3] Although Scriven explicitly equates "unstated assumptions" and "Missing premises" [p. 179], he never refers to the three "assumptions" of the homosexual argument as premises. Indeed, in none of his examples does Scriven use "missing premise" when referring to a patently untenable "unstated assumption."

[4] I am charging Scriven's account with much confusion. I want to add that I do find it valuable for its insights and illustrations, for its subtlety, and for distinctions made, if not observed.

[5] I am indebted to one of this journal's referees, Professor Robert Ennis, for trenchant criticisms of an earlier draft.

\section{references}

Ennis, Robert H., 'Identifying Implicit Assumptions," Synthese 51: 1, April 1982, pp. 61-86.

Hitchcock, David, Critical Thinking: a Guide to Evaluating Information, Methuen Publications, Toronto, 1983.

Johnson, Ralph H. "Charity Begins at Home," Informal Logic Newsletter 3:3, June 1981, pp. 4-9.

Schwartz, Thomas, The Art of Logical Reasoning, Random House, New York, 1980.

Schwartz, Thomas, "Logic as a Liberal Art,' Teaching Philosophy 4: 3 \& 4, July/October 1981, pp. 231-247.

Scriven, Michael, Reasoning, McGrawHill Book Company, New York, 1976.

van Eemeren, Frans $H$. and Grootendorst, Rob, "Unexpressed Premises: Part 1I," Journal of the American Forensic Association 19: 4, Spring 1983, pp. 215-225. $\square$

Professor Michael Burke, Department of Philosophy, Indiana University, 425 Agnes Street, Indianapolis, Indiana 46202 